\title{
Acción tutorial en el fortalecimiento del perfil profesional universitario: aportes en el desarrollo de competencias a partir de la educación virtual
}

\section{Tutorial action in strengthening the university professional profile: contributions in the development of competences from virtual education}

CHACON, Milagros D. ${ }^{1}$

\section{Resumen}

La acción tutorial en educación superior permite el desarrollo integral de los estudiantes mediante procesos orientados a la adquisición de competencias; en el contexto actual, la educación virtual se presenta con adaptación hacia nuevos escenarios, roles, metodologías y nuevos procesos de aprendizaje. Metodológicamente este artículo es de revisión donde se aplicó la búsqueda sistemática de información. Se concluye que las dimensiones más consideradas son en el área personal, y profesional, asimismo, se muestran las competencias del tutor.

palabras clave: desarrollo de competencias, educación virtual, acción tutorial

\begin{abstract}
Tutorial action in higher education allows the comprehensive development of students through processes aimed at acquiring skills; In the current context, virtual education is presented with adaptation to new scenarios, roles, methodologies and new learning processes. Methodologically, this article is a review article where the systematic search for information was applied. It is concluded that the dimensions most considered are in the personal area, and the professional also shows the skills of the tutor.
\end{abstract}

key words: skills development, virtual education, tutorial action

\section{Introducción}

En la actualidad la posición que ocupa la tutoría en la educación superior cobra real interés y relevancia desde el parecer de todos los participantes involucrados en esta acción. En el contexto del licenciamiento de las universidades en Perú, la Superintendencia de Educación Superior (SUNEDU), exige la tutoría como uno de los indicadores a evaluar ${ }^{2}$. Bajo esa disposición esta acción alcanza un nivel de consideración especial en los procesos evaluativos y en el desarrollo académico; influenciando el desarrollo de competencias, el rendimiento de las asignaturas y como espacio de mejora en la relación entre docentes y estudiantes.

\footnotetext{
${ }^{1}$ Universidad César Vallejo. Perú. ORCID: https://orcid.org/0000-0002-9757-1189 . email: milosha.b@gmail.com

${ }^{2}$ Ley Universitaria, N. (2014). 30220, en el capítulo VIII, Artículo 87, (87.5), menciona como deber del docente, "Brindar tutoría a los estudiantes para orientarlos en su desarrollo profesional y/o académico".
} 
Seguidamente, el desarrollo tecnológico actualmente tiene una gran influencia en la educación, y el uso de las Tecnologías de la Información y la Comunicación (TICS) en la educación virtual, digital, learning o en los sistemas de gestión de aprendizaje, las cuales permiten romper fronteras en el aula brindando la oportunidad de innovar en educación a través de nuevos escenarios, nuevos procesos y prácticas educativas.

En ese sentido, Piña y Torrealva (2020) consideran, que las tecnologías de la información a nivel universitario permiten innovadoras prácticas para el desarrollo de actividades académicas, propiciando la interacción, habilidades comunicativas, dinamismo en los contenidos en un mismo espacio, lo que influye en el adecuado desarrollo de los contenidos, acompañamiento y motivación.

La coyuntura actual por la pandemia por Corona Virus 2019 (COVID-19), causada por el virus SARS-CoV-2, declarada por la OMS; tuvo implicancia en la educación modificándose con ello los procesos de enseñanza aprendizaje. Britez (2020) menciona que docentes y estudiantes a nivel mundial tuvieron que involucrarse en el uso de las TICS de modo raudo, evidenciando el desarrollo de nuevas destrezas y habilidades. En ese sentido, la acción tutorial se representa como uno de los principales elementos que delimita la calidad de la formación en modalidad de educación virtual, brindando la posibilidad de orientación en cualquiera de los aspectos que pueden perjudicar el aprendizaje del estudiante por ello, Martínez, Pérez, González, González Lorente y Martínez (2020) resaltan, que existe la necesidad de analizar la respuesta de los estudiantes en esta acción, en relación a su desarrollo, evaluando las prioridades y deficiencias como nuevas propuestas de mejora que favorezcan el cumplimiento de los objetivos curriculares.

Sánchez y Gallego (2015) consideran que se requiere delimitar las técnicas y competencias más apropiadas para esta acción virtual y un análisis minucioso sobre los recursos que se disponen para este fin, ello adherido a las múltiples ventajas y estrategias que permiten el desarrollo en esta modalidad con el apoyo de la tecnología para responder a la formación integral del estudiante.

Teniendo en cuenta que el fomento de la tutoría académica es de carácter integral, donde ese pretende potenciar aprendizajes y competencias en el estudiante entorno a la educación virtual para lograr sus metas, se resalta las responsabilidades que esto implica, por ello se emprende este estudio donde el objetivo general es, explicar los principios orientadores de la acción tutorial en el desarrollo de competencias del perfil profesional a partir de la educación virtual. Asimismo, (a) reconocer las dimensiones de la acción tutorial que debe desarrollarse con el estudiante, (b) identificar las competencias tutoriales que fortalecen el perfil profesional del estudiante universitario, (c) describir las competencias del tutor en el desarrollo de las acciones tutoriales con los estudiantes y (d) explicar las estrategias de acción tutorial que podría implementar la universidad.

\section{Metodología}

Desde el punto de vista metodológico se asume el tipo de artículo de revisión, que es definida como la operación documental de rescatar un conjunto de referencias bibliográficas o documentos publicados en el mundo sobre un tema, una publicación, un autor o un trabajo específico. Asimismo, este tipo de trabajo a través de la revisión es clave para identificar áreas de investigación, nuevas tendencias; en ese sentido, se requiere de la realizar síntesis, disponer de fundamentación conceptual y teórica en relación de una disciplina y cómo evoluciona en el tiempo, demostrando su progreso. Junto a ello favorece la difusión del conocimiento científico y se mejoran las prácticas profesionales. (Guirao, 2015; Xu, Kang y Son, 2015)

Se consideran tres procesos importantes en este tipo de trabajo de investigación: la búsqueda de la información, organización y análisis de información. Como estrategia estos procesos favorecen tanto la obtención de documentación referente a un tema de investigación, así como sistematizarla y estructurarla con el objeto de analizar las principales características del conjunto de documentos bajo estudio. 
En búsqueda de la información se considera el uso fuentes primarias como revistas, tesis, monografías, libros y las fuentes secundarias que son resúmenes de bases de datos confiables y especializadas. La revisión de la literatura sigue una secuencia ordenada y metodológica también es la para el fundamento teórico; garantizando que el trabajo pueda tener un alto nivel científico. (Calle, L. F. 2016)

Con respecto a la organización de la información la técnica a utilizar será la del fichaje, que consiste en el uso de un instrumento en el que se plasma por escrito información científica importante, tras realizar el proceso de búsqueda de información. Organizar en esta modalidad la información, es más manejable unir ideas sobre un tema y obtener su ubicación con facilidad. Las fichas más utilizadas son: fichas bibliográficas, fichas de trabajo o de contenido y fichas de crítica a la fuente. (Castro, A., 2016)

El instrumento utilizado es la ficha sincrética, que permite la organización de la información. Los elementos de la ficha sincrética elaborada cuentan con la siguiente estructura: Título del contenido de la ficha, título de la monografía, tipo de ficha: resumen, textual o comentario; datos de localización de la fuente de información, contenido de la ficha, página de la monografía donde se encontrará el contenido de la ficha, sub capítulo, acápite o sub acápite de la monografía donde se encontrará el contenido de la ficha.

Imagen 1

Ficha sincrética

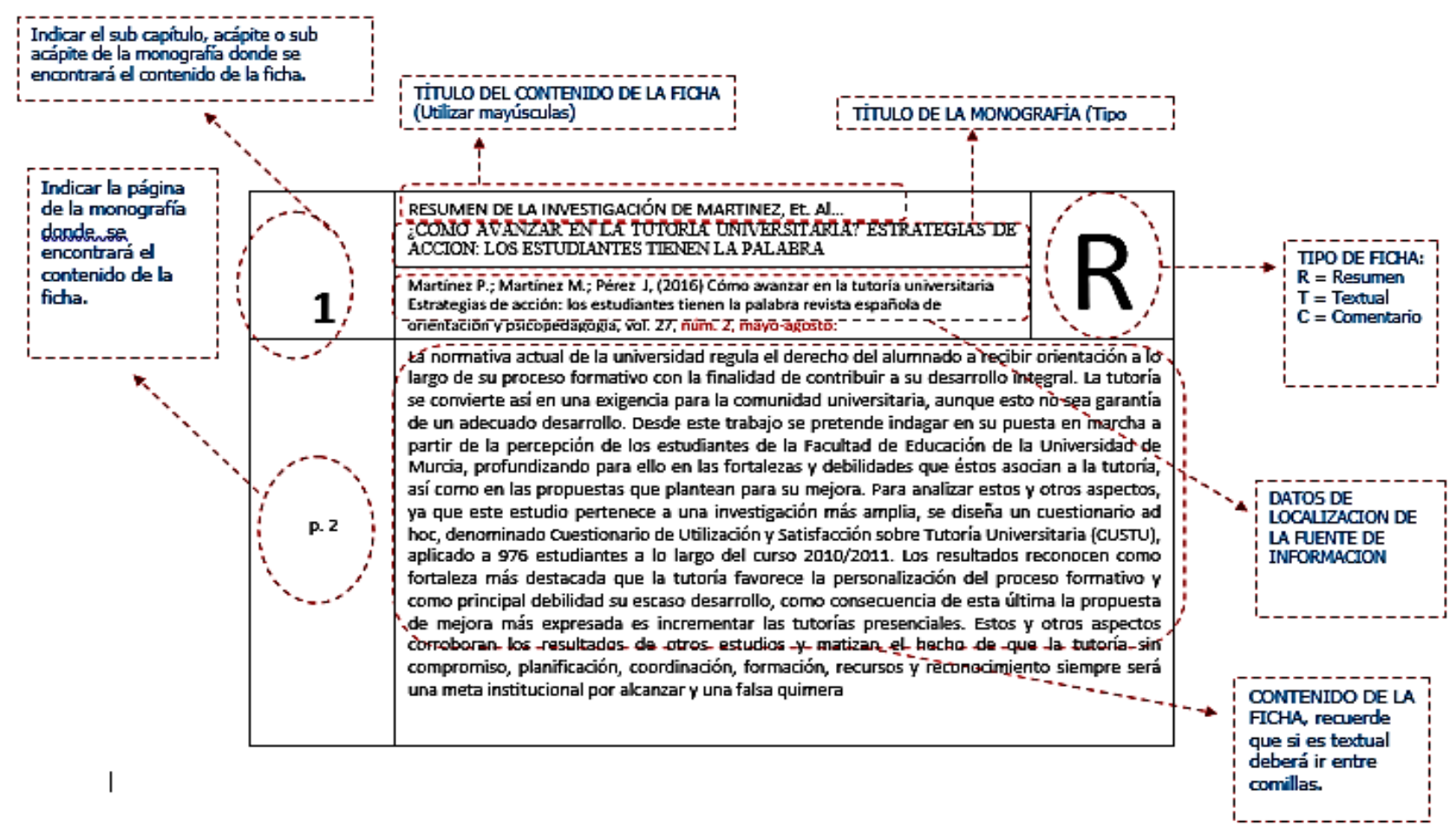

Como procedimiento para sistematizar la información se pudo trabajar con herramientas de búsqueda y sistematización de la información como Mendeley, asimismo se ubicaron documentos en las bases de datos como dialnet, ebsco host, google académico, redalyc, scielo, researchGate, revista espacios, etc. y biblioteca virtual de la UCV.

\section{Resultados y discusión}

La acción tutorial es aquella variable que equilibra, compensa, nivela o realimenta la función de un sistema formativo para la educación en la virtualidad, por ello Serrano, Pallares y Alonso (2020) expresan que promueve 
el interés, influye en el comportamiento, propicia el logro de metas y objetivos en el proceso de aprendizaje; en ese sentido desde esta acción, los entornos virtuales de aprendizaje son un nuevo enfoque para la apropiación y adquisición de las nuevas competencias educativas.

Esta actividad, como indica García, Rodríguez y Cáceres (2018) se presenta no solo como una acción de instruir al estudiante en el busca del desarrollo humano, en los aspectos académico, personal y profesional; a través de estrategias metodológicas en la educación tanto presencial como virtual, sino que buscan la implementación de competencias de acuerdo a cada programa con la intensión de evitar la deserción estudiantil, solucionar conflictos y ser punto de partida hacia nuevos enfoques.

De igual manera Alonso, Rodríguez y Cáceres (2018) describen que existe una destacada labor de la tutoría en relación a la atención de los requerimientos de los estudiantes siendo en clasificación las necesidades personales, académicas, profesionales, docentes y burocráticos, este último en menor medida. Asimismo, la acción tutorial tiene una influencia favorable para el seguimiento metódico activo y como mejorar las relaciones entre estudiantes y tutores.

Se considera una estrategia la acción tutorial virtual, porque permite un mayor acceso y uso por parte de los estudiantes; en ese sentido, promover estrategias de comunicación y de información en tiempo real, ya que el estudiante requiere atención fluida, rápida y personalizada. En ese sentido Saberbein (2018) menciona que existe una relación entre la educación virtual, aprendizaje colaborativo y la instrucción de estudiantes universitarios para la acción tutorial. A la vez indica que la formación de los estudiantes para ser tutores se relaciona con la enseñanza virtual referida a los aspectos formales, participación en foros, aportaciones y evidencias de aprendizaje.

Detrás de esta modalidad de educación, Esteban y Fuentes (2015) afirman que existe una gran responsabilidad, ya que puede afectar uno de los ejes que mueven la educación, el de cultivo de virtudes que forman de modo integral al estudiante, tanto en conocimiento, como en su desarrollo como persona y ser humano. Entonces es oportuno reconocer que considerar el avance tecnológico en educación, a través de la educación virtual, no asegura el logro del desarrollo humano; en ese sentido, se afirma que mantener el adjetivo humano en la esencia de la universidad sólo será posible en tanto no se transforme la autonomía de la relación educativa universitaria.

Tiempo atrás se consideraba que la dimensión tecnológica era muy importante para poder desarrollar esta acción de modo virtual pero actualmente Zapata (2017) describe que existe una disminución constante en la necesidad de esta acción ya que los estudiantes han adquirido el dominio en el uso de recursos digitales y de la accesibilidad a internet, por ello, las necesidades personales no influyen en la inasistencia de los estudiantes para recibir las sesiones tutoriales.

Cabe mencionar que Martín, Berrios, y Pantoja (2020) consideran que las dimensiones que intervienen en la tutoría, en áreas académica, personal y profesional dependen de componentes bien diferenciados para el adecuado desarrollo de esta acción, siendo el nivel de implicancia, asumir compromiso, demostrar experiencia, relación con el entorno profesional y habilidades interpersonales. Cabe mencionar que existe una dimensión administrativa que influyen transversalmente involucrando la dinámica de las instituciones, donde debe de presentarse con postura de colaboración y compromiso que refleje una planificación entre las derivaciones para las acciones, actitud colaborativa de todos los implicados, un sistema formativo efectivo y reconocimiento institucional.

La tutoría se ha contemplado a nivel mundial desde todos los ámbitos educativos y resaltando su importancia. Obaya y Vargas (2014) mencionan que esta acción muestra su aporte en el desarrollo integral del estudiante, por ello no se debe considerar a esta acción como un afianzamiento de un curso, tampoco solo para dar simple asesorías. Por el contrario, fomenta y favorece desde el pensamiento y evaluación de los estudiantes al 
desarrollo de habilidades académicas, así como en su desarrollo como persona y se plantea espacios de integración para el desarrollo de la acción tutorial.

El desarrollo de competencias en la formación, según Durán (2016), es uno de los requisitos para poder garantizar que se produce conocimiento, a través de destrezas, aptitudes y actitudes que generan los resultados de los estudiantes y bajo esta perspectiva, se asevera que con el desarrollo de las competencias de las personas se incrementa la calidad del conocimiento que éstas realmente alcanzan. Las competencias exigen un rediseño de los contenidos encaminados a formar personas capaces, estableciendo las bases y la profundización disciplinar que garanticen el desarrollo personal e intelectual, asi como una empleabilidad en concordancia con las demandas del mercado laboral y del bienestar de la sociedad.

Hoy en día, tomando como referencia a González Reyes, et al. (2018), las modificaciones a nivel global establecen un currículo basado en competencias hacia la profesionalización que integra las áreas curriculares y promueve nuevos procesos metodológicos de enseñanza que acerque al estudiante a la realidad profesional, por ello nos direccionamos a trabajar con enfoques integrales que busquen el saber conocer, saber ser, saber convivir, saber hacer y el saber transformarse para la sociedad; estimulando la autonomía, y eficiencia de los estudiantes hacia la gestión de los conocimientos y la información, logrando que el estudiante sea proactivo de su formación.

En ese sentido, Penalillo, Huerta, Huamani y Zanabria (2020) resaltan que, la tutoría virtual contribuye en el desempeño académico en educación superior y ante ello, Cala, Santos, y Pérez (2020) exponen las fortalezas del plan de acción tutorial virtual, aceptando que incrementa la atención diversa y es base para aumentar el éxito de los procesos de enseñanza, haciendo partícipes a los estudiantes y por ello se evidencia un elevado nivel de aceptación en el logro de las metas curriculares bajo esta modalidad.

Como consecuencia Navarro y Olmo (2015), mencionan que la concientización del desarrollo de competencias en estudiantes es fundamental, así mismo, el trabajo colaborativo, participativo, comunicación asertiva, apertura y confianza en consultar más en la modalidad de tutoría virtual, comprendiendo que el desarrollo de esta modalidad es necesario para lograr la integración y comprensión. Esta modalidad virtual según Durand (2015), tanto en pregrado como en post grado, muestra una aceptación favorable siendo esta modalidad un procedimiento que abona tanto competencias como aprendizajes en los estudiantes universitarios.

En relación a lo anterior, Marciniak (2016) describe que la educación virtual se presenta como un proceso interactivo y sistematizado y que requiere de recursos que motiven el aprendizaje promoviendo la autonomía en los alumnos, algunas características de esta modalidad son que propicia el auto estudio y la interacción simultanea; desarrolla la autonomía permitiendo la libertad y compromiso; promueve el desarrollo de estrategias comunicativas entre docentes y estudiantes a modo individual como grupal; compromiso en la planificación y la organización de la tutoría. Asimismo, cabe considerar la expresión de Vargas, Iniguez y Pichardo (2015), donde la adhesión a las demandas actuales en educación implica el desarrollar competencias integrales en los estudiantes teniendo en cuenta los tres saberes que forman al ser humano; el saber ser, el saber conocer y el saber hacer, para lograr ser competente en la sociedad actual, para hacer frente a los problemas de la realidad.

La tutoría según Díaz, Abascal, y Uscanga, (2019) actúa para restaurar conflictos cognitivos o afectivos, que se presentan en los estudiantes siendo necesario cuando se requiere llegar a un consenso, con la posibilidad de reestructurar los grupos y programaciones si fuese necesario. Para lograr la intervención cada participante dispondrá de destrezas a través de competencias comunicativas, ya que se debe cuidar esta dimensión con el objetivo de lograr el entendimiento entre ambos actores. Se resalta entonces la importancia de lograr intervenciones integrales que ayuden al acompañamiento del estudiante durante su formación universitaria. En ese sentido, la tutoría se presenta como mediador estratégico para propiciar su formación basado en su 
contexto. Cabe hacer mención que las tutorías presenciales en muchos casos se presentan más valoradas, pero la modalidad virtual en educación cobra cada vez mayor valor y se reconoce su utilidad y beneficios en los estudiantes, permitiendo el desarrollo de estrategias significativas en formato digital, tales como realizar discusiones en línea, integrar contenidos relevantes de la red o para posibilitar la participación de expertos o profesionales externos en los debates o charlas.

Con respecto a las competencias del tutor, se necesita identificar cuál es la acción más oportuna para solucionar un conflicto y saber ejecutarlo. Se asevera entonces que para ejercer la docencia es necesario desarrollar ciertas competencias, más aún cuando se tienen distintos contextos donde se está llevando a cabo la acción de enseñanza-aprendizaje debido a la inserción de las tecnologías. Esta nueva realidad obliga a que adicional a las competencias existentes en el docente universitario se requiere de otras competencias que lo presenten y formen como guía y orientador que no sólo transmita conocimientos a sus estudiantes, sino que fomente el aprendizaje de manera autónoma, aprovechando los recursos que se ofrecen TIC y espacios tecnológicos donde se efectúa la acción.

Por lo anterior Duran (2016), sostiene que a partir del uso de buenas prácticas educativas, considerando el uso de estrategias planificadas y con un adecuado diseño curricular en la educación virtual se logran resultados positivos. Cabe añadir las opiniones de Guerra, Lima y Lima (2017) quienes refieren que la tutoría tiene como influencia a la experiencia y dedicación del tutor, su formación académica, su compromiso como docente formador y el apoyo de la propia institución. Por ello, Aceves, Martinez y Barak (2019) mencionan que el tutor cumple un rol clave en la formación universitaria sobre todo en el acompañamiento del estudiante, por ello requiere de habilidades adecuadas donde detecte y atienda oportunamente bajo estrategias individuales 0 grupales. Por ello debe de capacitarse adquiriendo conocimientos metodológicos y estrategias para apoyar a los estudiantes.

En ese sentido Montoya, Montoya y Yanza (2020) comentan que existe una diferencia importante en la enseñanza durante los periodos evaluativos gracias a las tutorías virtuales, por ello es importante el trabajo de la acción tutorial académica, donde el tutor debe demostrar habilidades, destrezas, competencias, valores y estar actualizado en el manejo de la tecnología para un óptimo desempeño. Por tanto puede decirse que esta acción, según Oliva et al.(2020), favorece la mejora de las funciones técnicas, académicas, organizativas, orientadoras y sociales de los tutores virtuales. Todo ello permite hacer una valoración al uso de estrategias comunicativas, seguimiento, refuerzo pedagógico, y la evaluar los aprendizajes puede ayudar a mejorar la tutoría virtual.

El tutor virtual, según aseveran Antón y López (2020) se presenta como una figura poliédrica que acompaña al estudiante en su proceso de formación facilitando la comprensión de los contenidos, estimulando el aprendizaje y retroalimentando, para animar a la mejora y a la obtención de los logros curriculares programados, por ello desde el punto de vista de los estudiantes, Alves, Simão y Leitinho (2018) mencionan que el tutor tiene que ser proactivo, que brinde una actitud empática y se encuentre dispuesto a atender las diversas necesidades que se requieren.

Para Cuadrado et al. (2020), la tutoría en la enseñanza virtual otorga características propias al proceso tutorial, por ello se asevera que en la enseñanza a distancia se revelan necesidades tutoriales específicas que demandan un perfil competencial propio y diferencial en el docente y tutor, siendo conveniente un proceso de selección e instrucción previa para el éxito de esta modalidad educativa. Pero en la actualidad según sostiene Cariaga (2020) algunos docentes no se adaptan a la exigencia de nuevas estrategias comunicativas y de nuevas formas de producir conocimiento. En ese sentido las entidades educativas deben gestionar, replantear roles docentes y estimular políticas de desarrollo profesional para que permitan a las TIC como una oportunidad en el desarrollo de competencias. 
Cabe considerar que, en educación universitaria, sobre todo en carreras de educación es importante formar a los futuros educadores en estrategias tutoriales, en ese sentido el comentario de Martínez y Martínez (2015) aseguran que desarrollar un programa de tutoría en pequeños grupos, como estrategia es una opción en carreras de educación porque ayuda a aclarar los métodos pedagógicos para apoyar a la formación en educación superior que estén a la par con la educación europea.

Son importantes las expresiones de Martin y Martínez (2016) donde afirman que mientras exista educación enfocada en el alumnado, el desarrollo de nuevas pedagogías y el rol de la universidad en la sociedad actual son componentes que requieren alternativas pedagógicas que faciliten la interacción por medio de la tutoría, no obstante se ignora la evaluación y apreciación que tienen estudiantes universitarios de los programas de educación frente a la acción tutorial académica, direccionada al acompañamiento. En ese sentido la tutoría presencial, según Carranza et al. (2020) tiene mejor aceptación por los estudiantes, a diferencia de la modalidad virtual, esto puede ser resultado por la carencia de un diseño instruccional y falta de motivación. Asimismo, es importante mencionar que la tutoría virtual ayuda en el proceso de aprendizaje, siempre que exista estrategias motivadoras y bien planificadas, es decir el acto de la motivación resulta ser un factor indispensable en esta modalidad de educación en línea.

El uso de plataformas digitales es considerado por García y Casal (2020) útil para la realización de seguimiento y tutoría, pero aún existe una preferencia por la tutoría presencial y a la ves, el desconocimiento de nuevas posibilidades de esta herramienta. Con ello se evidencia que se necesita compromiso y la motivación necesaria para el desarrollo de esta modalidad. En ese sentido Díaz y Colorado (2020) sugieren como recurso al uso de plataformas digitales como Moodle, ya que posee características funcionales para el desarrollo de la acción tutorial de modo eficaz y ayuda con el diseño, implementación e integración curricular, y de competencias tecnológicas tanto en docentes como estudiantes.

Lo anterior también se puede vincular con lo expresado por Ameli et al. (2019), donde refieren que un programa de tutorías virtual puede presentar debilidades si existe falta de compromiso de los estudiantes, falta de guía o instructivo de contenidos y procedimientos que unifiquen criterios de los tutores virtuales, falta de concientización de los tutores y estudiantes en el empleo de diversas herramientas de tutoría virtual con un sentido humanístico y de pertenencia por medio de la creación de escenarios virtuales que permitan aproximar al alumno tutorado con el docente tutor y la institución.

Considerando lo importante de la mención anterior, Oliva et al. (2020) comentan que en un programa de tutoría virtual si se trabaja de modo activo y participativo con las actividades programadas, logran influenciar en la estructura, en la programación y en la adquisición de destrezas tecnológicos en estudiantes y tutores, afianzando así los roles de los protagonistas de esta acción permitiendo lograr los objetivos curriculares.

Una representación de estrategia es lo sugerido por Castillo, Zorrilla y Acosta (2019) donde proponen la implementación de cubículos virtuales con el objetivo de acompañar y reforzar cada etapa formativa del estudiante. Se puede señalar que es necesario la motivación a través de estrategias para propiciar la participación de los estudiantes, asimismo se sugiere categorizar las actividades por etapa formativa: general, disciplinar y profesional, además de incluir otras actividades complementarias para brindar al tutor un abanico de opciones que cubran las necesidades de su tutorado.

Asimismo, es conveniente según Bedregal (2019), programar acciones individuales y cooperativas que apoyen al desarrollo de competencias, intensifican la motivación por la asignatura y permiten la creación de redes académicas y profesionales. Sumado a ello, los estudiantes reconocen el uso de plataformas digitales como una adaptación rápida, que facilita la comunicación entre pares y con el tutor, evitando así inconvenientes. 
Estas estrategias se asemejan a lo planteado por Basantes, Naranjo y Ojeda (2018) que proponen el método PACIE (Presencia, Alcance, Capacitación, Interacción, E-learning) desarrollado en entornos digitales de aprendizaje y que favorece considerablemente la programación curricular virtual, el trabajo colaborativo, eleva la calidad, teniendo presente el sentido de humanismo en el desarrollo de esta estrategia, y demuestra gran aceptación, evidenciándose esto en las percepciones y valoraciones de los estudiantes.

Los análisis de Aguilar et al. (2015) mencionan que los docentes de ciencias de la educación y salud son los más capacitados y muestran compromiso para el desarrollo de las competencias en tutoría, con ello se quiere expresar que es necesario incrementar planes y programas dirigidos a la formación del profesorado con respecto a la función tutorial. Finalmente, Hernández et al. (2020) afirman que un ejemplo de educación disruptiva es considerar la inclusión de nuevas tecnologías de la información en el proceso de aprendizaje, sesiones tutoriales personalizadas y flexibilidad en los horarios, como estrategia de mejora en el rendimiento académico en la universidad a través de la implementación de la tutoría virtual.

\section{Conclusiones}

En la educación actual, a modo de demostrar la calidad educativa, es importante para la formación integral de los estudiantes en la modalidad de educación virtual, un tipo de apoyo y orientación permanente con mucho más empeño y que se presente no solo a dar cumplimiento a una planificación, sino que se disponga del compromiso de cada actor involucrado en esta acción, que aun presenta ciertas fisuras educativas que influye en su cumplimiento. En ese sentido, la acción tutorial se presenta como un elemento indispensable en el fortalecimiento del perfil profesional universitario contribuyendo al desarrollo de competencias a partir de la educación virtual.

En ese sentido se concluye que las dimensiones trabajadas en la modalidad de educación virtual son: la dimensión académica, personal, y profesional, en busca del desarrollo humano, siendo muy importantes en la educación virtual donde se evidencian diversas necesidades estudiantiles y donde la universidad debe asumir el compromiso de formación integral con sus estudiantes.

Lass competencias desarrolladas en los estudiantes que reciben la acción tutorial logran perm iten su desarrollo integral, que involucra el desarrollo del pensamiento, de la persona, el trabajo participativo, habilidades comunicativas, capacidad de apertura, confianza y desarrollo de autonomía; siendo muy importante crear la mejor planificación de la acción tutorial para que se cumplan los objetivos y se logre el desarrollo de estas competencias.

Las competencias del docente demostradas en la acción tutorial virtual son: competencias digitales por parte del docente y del alumno, para hacer el uso adecuado de las TIC. Asimismo, el desarrollo de habilidades comunicativas demostrando experiencia y dedicación en la obtención de logros.

Como estrategias de acción tutorial en el proceso formativo universitario virtual, se requiere de la instrucción y concientización previa por parte de todos los actores involucrados. Así mismo, se propone el desarrollo en pequeños grupos, aplicando elementos motivadores y bien diseñados. El uso de recursos digitales es indispensable para garantizar la adecuada conducción de la tutoría y con ello demostrar y propiciar, el trabajo activo y participativo.

Los cubículos virtuales se presentan como una estrategia importante para reforzar la etapa formativa del estudiante, con estos, a través de acciones individuales y cooperativas, se propicia el trabajo colaborativo afianzando las destrezas interpersonales.

Se resalta la importancia de lograr intervenciones integrales que ayuden al acompañamiento del estudiante durante su formación universitaria. En ese sentido, la tutoría se presenta como mediador estratégico para propiciar su formación, basado en su contexto. Por ello, se recomienda el desarrollo de trabajos de investigación aplicativos, basados en programas de tutoría universitaria en la modalidad virtual. 


\section{Referencias bibliográficas}

Aceves, Y.; Martinez, Y. y Barak, M. (2019) Necesidades formativas en tutores universitarios del área de psicología. Espacios. Volumen 40 (18), página 14. Recuperado de: http://www.revistaespacios.com/a19v40n26/a19v40n26p23.pdf

Aguilar, J.; Alias , A.; Álvarez, J.; Fernández, J.; Pérez, E. y Hernández, A. (2015). Training needs of university professors in skills related to the tutoring action ; Necesidades de formación del profesor universitario en competencias relacionadas con la acción tutorial. Recuperado de: https://doi.org/10.4995/redu.2015.5433

Aguilera ,R.(2014). ¿Revisión sistemática, revisión narrativa o metaanálisis?. Revista de la Sociedad Española del Dolor, 21(6), 359-360. Recuperado de: https://dx.doi.org/10.4321/S1134-80462014000600010

Alonso,S.; Rodríguez, A. y Cáceres M. (2018). Análisis de la Acción Tutorial y su Incidencia en el Desarrollo Integral del Alumnado. El Caso de la Universidad de Castilla La Mancha, España. Formación Universitaria, 11(3), 63-72. Recuperado de: https://doi.org/10.4067/S0718-50062018000300063

Alves, F.; Simão, A. y Leitinho, M. (2018). Acción tutorial en la enseñanza superior: experiencia con alumnos mayores de 23 en una institución pública de Portugal. Educação \& Formação, 3(7), 44-65. Recuperado de: https://dialnet.unirioja.es/servlet/articulo?codigo=7146549

Ameli ,G.; Martínez ,C.; Serrano ,A.; Espinal ,D. y Figueroa, D. (2019).EL PROBLEMA DE LA TUTORÍA VIRTUAL EN UNA UNIVERSIDAD PÚBLICA EN EL SUR DE MÉXICO. INCLUSIÓN EDUCATIVA Y RESPONSABILIDAD SOCIAL, 7. Recuperado de: https://www.uv.mx/tutorias-anuies/files/2020/03/Capitulo-4.pdf\#page=9

Antón, J. y López, A. (2020). Claves para la tutoría virtual. Revista Saberes Educativos, (5), 37-45. doi:10.5354/2452-5014.2020.57782. Recuperado de: https://sabereseducativos.uchile.cl/index.php/RSED/article/view/57782

Basantes, A.; Naranjo, M. y Ojeda, V. (2018). Metodología PACIE en la Educación Virtual: una experiencia en la Universidad Técnica del Norte. Formación Universitaria, 11(2), 35-44. Recuperado de: https://doi.org/10.4067/s0718-50062018000200035

Bedregal, N. (2019). Tutoría virtual y blended-learning en el posgrado: Orientaciones y resultados de una experiencia. En Actas de la Multi-conferencia internacional LACCEI para Ingeniería, Educación y Tecnología (Vol. 2019-julio). Consorcio Latinoamericano y del Caribe de Instituciones de Ingeniería. Recuperado de: https://doi.org/10.18687/LACCEI2019.1.1.220

Cala ,M.; Santos ,E. y Pérez, K. (2020). Importancia del Plan de Acción Tutorial en la Educación a Distancia. Revista de Investigación, Formación y Desarrollo: Generando Productividad Institucional, 8(1), 10-10. Recuperado de: http://ojs.formacion.edu.ec/index.php/rif/article/view/189

Calle ,L. (2016). Metodologías para hacer la revisión de literatura de una investigación. Recuperado de : https://www.researchgate.net/publication/301748735_Metodologias_para_hacer_la_revision_de_literatu ra_de_una_investigacion

Castro, A.(2016) Elaboración de fichas. U.D. de Investigación I.Recuperado de: https://investigar1.files.wordpress.com/2010/05/elaboracic3b3n-de-fichas.pdf 
Castillo ,M.; Zorrilla M. \& Acosta ,J. (2019). Implementación de la tutoría en la Licenciatura en Psicología, modalidad virtual. Apertura (Guadalajara, Jal.), 11(2), 54-71. Epub 25 de febrero de 2020. Recuperado de: https://doi.org/10.32870/ap.v11n2.1659

Cariaga, R. (2020). El rol del docente universitario en las propuestas educativas virtualizadas. Revista Pilquen. Sección Psicopedagogía, 16(2), 14-28. Recuperado de : http://revele.uncoma.edu.ar/htdoc/revele/index.php/psico/article/view/2547

Carranza, M.; Plascencia, R.; Islas ,C. \& Jiménez, A.(2020). Percepción de estudiantes universitarios sobre la tutoría presencial y la virtual: Perception of university students about face-to-face and virtual tutoring. EDUCATECONCIENCIA, 27(28), 126-146. Recuperado de: http://tecnocientifica.com.mx/educateconciencia/index.php/revistaeducate/article/view/259

Carrillo, D.(2014). Competencias TIC de los Docentes para la Enseñanza mediante Entornos Virtuales en Educación Superior. El caso de la Universidad de Los Andes-Venezuela: Evaluación y Diseño de un Plan de Formación . Recuperado de: http://hdl.handle.net/10803/285330

Cuadrado, A.; González, R.; Méndez, L. \& Malik, B. (2020). Competencias Tutoriales De Los/As Responsables De Prácticas Profesionales en Contextos De Enseñanza a Distancia. Revista Prisma Social, 28, 176-200. Recuperdo de: http://eds.a.ebscohost.com/eds/pdfviewer/pdfviewer?vid=3\&sid=017ad847-7a1d-4bf9ae6d-f00985de2fd3\%40sessionmgr4006

Diaz, M. y Colorado, B.(2020). Estudio para realizar la acción tutorial a través de un sistema de gestión de aprendizaje en Moodle para el nivel de secundaria. MLS Educational Research (MLSER), 4(1), 41-56. Recuperado de: https://doi.org/https://doi.org/10.29314/mlser.v4i1.260

Duran, R.(2016) La Educación Virtual Universitaria como medio para mejorar las competencias genéricas y los aprendizajes a través de buenas prácticas docentes, Universitat Politècnica de Catalunya. Recuperado de: https://www.tdx.cat/handle/10803/397710

Durán, R.; Estay, C. y Álvarez, H.(2015). Adopción de buenas prácticas en la educación virtual en la educación superior. Aula Abierta, 43(2), 77-86. Recuperado de: https://doi.org/10.1016/j.aula.2015.01.001

Esteban, F. y Fuentes, J. (2015). La educación universitaria virtual: una reflexión en torno al cultivo de las virtudes. TeoríA De La EducacióN. Revista Interuniversitaria, 27(1), 103-118. Recuperado de : https://revistas.usal.es/index.php/11303743/article/view/teoredu2015271103118/13439

García, B. y Casal , L.(2020). El uso del campus virtual para la tutorización y seguimiento del trabajo fin de grado: valoración del profesorado y propuesta de formación. Revista Iberoamericana De Educación Superior, 11(32), 216-227. Recuperado de: https://doi.org/10.22201/iisue.20072872e.2020.32.823

González, Y.; González, S.; Guerrero, D.; Gavilanes, F.; Balseca, J. y De los Ángeles, M. (2018). El aprendizaje autónomo. Su rol en el desarrollo de competencias específicas en la educación superior. (Spanish). Dilemas Contemporáneos: Educación, Política y Valores, 5(3), 1-19. Recuperado de: https://www.dilemascontemporaneoseducacionpoliticayvalores.com/index.php/dilemas/article/view/227

Guirao, J.(2015). Utilidad y tipos de revisión de literatura. Ene, 9(2). Recuperado de: https://dx.doi.org/10.4321/S1988-348X2015000200002

Guerra, M.; Lima , M.; y Lima, J. (2017). Oferta y uso de tutorías virtuales y presenciales: Percepciones de profesores y estudiantes de. Cultura y Educación , 29 (4), 798-832. Recuperado de: https://doi.org/10.1080/11356405.2017.1382047 
Hernández, J.; Avendaño, V.; Buitrón, H. y Castillo, V.(2020). Tutoría virtual como elemento disruptivo en el modelo ejecutivo de las Universidades Tecnológicas. Boletín Científico De La Escuela Superior Atotonilco De Tula, 7(14), 27-33. Recuperado de: https://doi.org/10.29057/esat.v7i14.6039

Ley Universitaria, N. (2014). 30220. Lima, Perú. Recuperado de: http://www.minedu.gob.pe/reformauniversitaria/pdf/ley_universitaria.pdf

Martín, A.; Berrios, B. y Pantoja, A. (2020). Factores y elementos de calidad percibidos por el profesorado participante en el plan de acción tutorial de universidades europeas. Educacion XX1, 23(1). Recuperado de:

https://www.researchgate.net/profile/Antonio_Pantoja_Vallejo/publication/340601338_FACTORES_Y_EL EMENTOS_DE_CALIDAD_PERCIBIDOS_POR_EL_PROFESORADO_PARTICIPANTE_EN_EL_PLAN_DE_ACCION_ TUTORIAL_DE_UNIVERSIDADES_EUROPEAS/links/5e94472e92851c2f529c39d3/FACTORES-Y-ELEMENTOSDE-CALIDAD-PERCIBIDOS-POR-EL-PROFESORADO-PARTICIPANTE-EN-EL-PLAN-DE-ACCION-TUTORIAL-DEUNIVERSIDADES-EUROPEAS.pdf

Martínez, V. y Martínez, Á. (2016). La acción tutorial académica en pequeños grupos: Una experiencia educativa con estudiantes universitarios. Revista Electrónica Educare, 20 (2), 1-26. [Fecha de consulta 11 de junio de 2020]. Recuperado de: https://www.redalyc.org/articulo.oa?id=1941/194144435022

Martínez , P. ; Pérez, J. ; González, N.; González, C. y Martínez , M. (2020). La Tutoría universitaria vista por sus alumnos: Propuestas de mejora. Revista De La Educación Superior, 49(195), 55-72. Recuperado de: http://189.254.1.230/ojs/index.php/resu/article/view/1251

Marciniak, R. (2016). Autoevaluación de programas de educación universitaria virtual. Universitat Autònoma de Barcelona, 2016. Recuperado de: http://eds.b.ebscohost.com/eds/detail/detail?vid=3\&sid=629de784febc-4016-b538-d266c69d5a0e\%40pdc-v sessmgr02\&bdata=Jmxhbmc9ZXMmc2I0ZT1/ZHMtbGI2ZQ\%3d\%3d\#AN=edstdx.10803.400023\&db=edstdx

Britez, M. (2020) La educación ante el avance del COVID-19 en Paraguay. Comparativo con países de la Triple Frontera Universidad Nacional del Este. Recuperado de: https://doi.org/10.1590/SciELOPreprints.22 https://preprints.scielo.org/index.php/scielo/preprint/view/22/version/417

Montoya, E. ; Montoya, J. y Yanza, W. (2020) Las tutorías académicas virtuales y el rendimiento académico de los estudiantes universitarios. Recuperado de: https://easychair.org/publications/preprint_download/z7vg

Navarro, J. y Olmo, F. (2015). Desarrollo de competencias transversales mediante tutoría virtual. Editorial Universitat Politècnica de València. Recuperado de: https://doi.org/10.4995/INRED2015.2015.1586

Obaya, A. y Vargas, Y. (2014) Tutoría en Educación Superior. Educación química , 25 (4), 478-487. Recuperado el 11 de junio de 2020. Recuperado de:

http://www.scielo.org.mx/scielo.php?script=sci_arttext\&pid=S0187893X2014000400012\&lng=en\&tIng=en

Oliva, L.; García, J.; Ruiz, M.; Borges, B.; Amado, H. y García, A. (2020). Herramientas para potencializar la tutoría virtual: una experiencia e-learning para el profesorado. Recuperado de: http://biblioteca.galileo.edu/tesario/handle/123456789/955

Penadillo, L.; Huerta, R.; Huamaní G. y Zanabria, P. (2020). Tutoría Virtual y Desempeño Académico en los Estudiantes de la Escuela Profesional de Educación de la Facultad de Ciencias Sociales, Educación y 
Comunicación; Universidad Nacional Santiago Antúnez de Mayolo. Recuperado de:

https://doi.org/10.32911/as.2011.v4.n2.542

Piña, E. y Torrealba, L. (2020). Los entornos virtuales de aprendizaje (EVA). Una Estrategia didáctica para la administración de unidades curriculares universitarias. Revista Electrónica De Divulgación De Metodologías Emergentes En EI Desarrollo De Las STEM, 1(2), 18-27. Recuperado de: http://www.revistas.unp.edu.ar/index.php/rediunp/article/view/119

Saberbein, J. (2018). Enseñanza estratégica virtual, aprendizaje colaborativo y formación de tutores en Orientación Vocacional en la Facultad de Tecnología de la Universidad Nacional de Educación, 2016. Recuperado de: http://repositorio.une.edu.pe/handle/UNE/2163

Sánchez, J.; Arce, L. y Rodríguez, A. (2020). Enfermedad por coronavirus 2019 (COVID-19) en América Latina: papel de la atención primaria en la preparación y respuesta. Recuperado de: https://dialnet.unirioja.es/servlet/articulo?codigo=7423799

Serrano, M. ; Pallares, M. y Alonso, M. (2020). Utilidad de las tutorías académicas en la universidad. Resultados agregados de un estudio multidimensional en diferentes ciencias. Revista de Ciencias de la Comunicación e Información, 25(1), 57-74. Recuperado de: https://dialnet.unirioja.es/servlet/articulo?codigo=7514333

Vargas, I.; Iniguez, F. y Pichardo, C. (2015). Development of Comprehensive Competences with Information and Communication Technologies in Distance Learning Higher Education/Desenvolvimento de competencias integrais com tecnologias da informacao e da comunicacao em educacao superior a distancia/DESARROLLO DE COMPETENCIAS INTEGRALES CON TECNOLOGIAS DE LA INFORMACION Y DE LA COMUNICACION EN EDUCACION SUPERIOR A DISTANCIA. Panorama, 16, 9 . Recuperado de: http://eds.a.ebscohost.com/eds/pdfviewer/pdfviewer?vid=2\&sid=94a9657a-706e-408e-9263$65 \mathrm{e} 486 \mathrm{~d} 77 \mathrm{ccf} \% 40$ sdc-v-sessmgr03

Xu, J.; Kang, Q. y Song, Z. (2015). The current state of systematic reviews in library and information studies. Library \& Information Science Research, 37(4), 296-310. Recuperado de: https://doi.org/10.1016/j.lisr.2015.11.003

Esta obra está bajo una Licencia Creative Commons Attribución-NoCommercial 4.0 International

\section{(cc) EY-NC}

